\title{
Readministration of Platinum Agents in Recurrent Ovarian Cancer Patients Who Developed Hypersensitivity Reactions to Carboplatin
}

\author{
CHIKAGE NARUI, HIROSHI TANABE, JASON S. SHAPIRO, YOKO NAGAYOSHI, \\ TAKENORI MARUTA, MOMOKO INOUE, YUKIHIRO HIRATA, HIROMI KOMAZAKI, \\ HIROKUNI TAKANO, SHIGEKI NIIMI, SEIJI ISONISHI and AIKOU OKAMOTO
}

Department of Obstetrics and Gynecology, The Jikei University School of Medicine, Tokyo, Japan

\begin{abstract}
Background/Aim: Hypersensitivity reactions (HSRs) to carboplatin, a key drug for ovarian cancer patients, are problematic. The aim of this study was to evaluate the efficacy and safety of readministration of platinum agents (PTS) in recurrent ovarian cancer patients who developed HSRs to carboplatin. Patients and Methods: Thirty-one patients with recurrent ovarian cancer who developed HSRs to carboplatin were divided into those who continued to receive PTs in the following cycle (continuation group, $n=24)$ and those in whom either the drug was switched to non-platinum agents (non-PTs) or chemotherapy was ended (discontinuation group, $n=7$ ). Outcomes were evaluated based on patients' medical records. Results: The median survival time following HSRs was 28.1 and 15.4 months in the continuation and discontinuation groups, respectively $(p=0.018)$. In the continuation group, a total of 155 cycles of PTs were re-administrated, and 50 cycles (32\%) led to recurrent HSRs. There were no recurrent HSRs with a severity of grade 3 or greater. Conclusion: Continuation of PTs in ovarian cancer patients may contribute to improvement in their overall survival without severe recurrent HSRs.
\end{abstract}

An estimated 295,000 patients developed ovarian cancer in 2018, with the disease ranking 8th among female cancers and accounting for about $3.4 \%$ of all cancers world-wide.

This article is freely accessible online.

Correspondence to: Hiroshi Tanabe, Department of Obstetrics and Gynecology, The Jikei University School of Medicine, 3-25-8 Nishi-Shimbashi, Minato-ku, Tokyo 105-8461, Japan. Tel: +81 334331111, Fax: +81 354738421, e-mail: htanabe@jikei.ac.jp

Key Words: Carboplatin, hypersensitivity reactions, ovarian cancer, rechallenge, overall survival.
The number of deaths from ovarian cancer is estimated to be 185,000 , which makes the disease the 8th leading cause of cancer-related death and indicates a generally poor prognosis (1). Platinum-based drugs are the most effective agents for patients with ovarian cancer when the primary lesion is located in the ovarian epithelium, fallopian tube, or peritoneum. Over the past two decades, cyto-reductive surgery followed by combination chemotherapy with platinum agents (PTs) and taxanes has become the gold standard of treatment for ovarian cancer $(2,3)$.

About $70 \%$ of patients with advanced ovarian cancer respond to chemotherapy with a PT, but recurrence is seen in most patients. Following recurrence, chemotherapy regimens, including $\mathrm{PTs}$, are mainly used with the aim of prolonging life, provided that the patient is sensitive to these agents (4). Carboplatin is most widely used agent because of its low toxicity and ease of administration. However, the development of hypersensitivity reactions (HSRs), which include symptoms such as rushes, itching and sometimes breathing difficulties, are problematic $(5,6)$. Moreover, it is said that the incidence of these reactions increases from a small amount to more than $15 \%$ when more than 6 cycles are administered (7). Since the median number of cycles at the initial HSR (iHSR) ranges from 6-8, HSRs mainly develop after second-line treatment following recurrence in many cases. Once an HSR to carboplatin develops, it recurs in many cases despite the use of preventive measures, such as premedication $(6,8,9)$. Thus, treatment with PTs is abandoned in many cases, despite the fact that these agents are key therapeutic drugs. This phenomenon could potentially have a severe negative impact for recurrent ovarian cancer patients with poor prognosis.

Readministration of carboplatin following the development of an HSR requires increased dose of antiinflammatory medication or desensitization using multistep dilution or extended infusion (10-12). A switch to PTs, such as cisplatin and nedaplatin has also been examined $(7,13)$. 
In this setting, however, it is unclear whether continuation of PTs post iHSR has a greater effect on prognosis compared to withdrawal of these agents. Therefore, the goal of this study was to investigate the efficacy and safety of readministration of PTs in patients with ovarian cancer following the development of an iHSR to carboplatin, using a retrospective review of medical records.

\section{Patients and Methods}

Subjects. Of 530 patients who were histo-pathologically diagnosed with ovarian cancer with the primary lesion in the ovarian epithelium, fallopian tube, or peritoneum at our four related facilities between 2008-2012, recurrent ovarian cancer patients who developed iHSRs to carboplatin during second-line or more chemotherapy were retrospectively evaluated based on their medical records. Eligible patients were regarded as platinum-sensitive and were scheduled to continue to receive platinum-based chemotherapy at the time of iHSRs. Patients with platinum-sensitive recurrence who received platinum agents with second-line regimens or additional chemotherapies following first-line treatment with a carboplatin and paclitaxel were included. Patients who developed iHSR to carboplatin during the first-line chemotherapy as an initial treatment were excluded. Patients who had reason to discontinue platinumbased chemotherapy at the occurrence of iHSRs, such as disease progression, were also excluded. Eligible patients were divided into two groups based on the treatment course after development of iHSR: i) those who received regimens with PTs in the following cycle (continuation group), and ii) those for whom the treatment was switched to regimens with non-platinum agents (non-PTs) in the following cycle or chemotherapy was ended (discontinuation group). PTs included: i) carboplatin, ii) cisplatin, and iii) nedaplatin. NonPTs included all chemotherapeutic agents except PTs. Regardless of the regimen contents, each cycle administration, including PTs, was counted for the numbers of cycles of PTs and each cycle administration without PTs was counted for the numbers of cycles of non-PTs (Figure 1). Patients' background, timing and severity of iHSRs, chemotherapies after iHSRs, and overall survival (OS) were investigated. The course of the continuation group was analyzed based on preventive measures and outcomes in readministration of PTs following the development of iHSRs.

Criteria for HSRs to carboplatin. HSRs were defined as allergic reactions that included rash, itching, facial flush, chest tightness, breath difficulty, emesis, abdominal pain, diarrhea, hypotension, hypertension, tachycardia and bradycardia. HSRs caused during or at the end of the carboplatin infusion were included. HSRs caused during infusion of other agents or after a few hours from carboplatin infusion were excluded because they were possibly irrelevant to carboplatin.

Severity of HSRs. The reaction severity was evaluated using the allergic reaction category of the Common Terminology Criteria for Adverse Events (CTCAE ) ver. 4.0 (13): i) grade 1 (G1): transient flushing or rash, drug fever $<38^{\circ} \mathrm{C}\left(<100.4^{\circ} \mathrm{F}\right)$, intervention not indicated; ii) grade $2(\mathrm{G} 2)$ : intervention or infusion interruption indicated, responds promptly to symptomatic treatment, prophylactic medication indicated for $\leq 24$ hours; iii) grade 3 (G3): prolonged, recurrence of symptoms following initial improvement, hospitalization indicated for clinical sequelae; iv) grade 4 (G4): lifethreatening consequences, urgent intervention indicated; and v) grade 5 (G5): death.

Statistical analyses. OS was defined as the time from iHSR until death as a result of any cause, and patients alive at the time of the analysis were censored at the date of last contact. To identify independent prognostic factors, known prognostic factors and factors affecting the administration of PTs after iHSR were analyzed.

Continuous variables were analyzed using the unpaired Student's $t$-test or Mann-Whitney $U$-test. Categorical variables were analyzed using the Fisher's exact test. OS was evaluated using the Kaplan-Meier method and compared by log-rank test. Prognostic factors were analyzed using the Cox proportional hazard regression model (multivariate analysis). Values of $p<0.05$ (two-sided) were regarded as significant in all analyses. All calculations were performed using the statistics software R, version 3.5.1.

\section{Results}

Thirty-one patients who developed HSRs to carboplatin were included. The continuation and discontinuation groups included 24 and 7 patients, respectively. The treatment course following the development of iHSRs in the discontinuation group was switching to non-PT-based chemotherapy. One patient never restarted any PTs, and discontinuation of all chemotherapy occurred in one other patient only. The other 5 patients in the discontinuation group restarted PTs after several cycles with non-PTs because they developed disease progression during the use of non-PTs. The median follow-up was 24.1 months (range $=1.7-71.6$ ) for patients with censored data. At that time, 27 patients were dead and 4 patients were alive with disease. All 4 patients who were alive during the final follow-up were from the continuation group.

Patient characteristics. The patients' characteristics in the continuation and discontinuation groups are shown in Table I. The median number of carboplatin cycles at iHSR that was summed from the first cycle of first-line chemotherapy to the cycle of iHSR were 10 (range=7-17) in the continuation group and 12 (range=9-16) in the discontinuation group. The severity of the allergic reaction was $\mathrm{G} 1$ in $8(33 \%)$ and 0 $(0 \%)$, and $\mathrm{G} 2$ in $16(67 \%)$ and $7(100 \%)$ cases, respectively. No reaction of G3 or greater occurred in either group. The patients' backgrounds and demographic characteristics were not significantly different between two groups.

Treatment administration after iHSR. Chemotherapy regimens following iHSRs are shown in Table II. The median number of cycles of PTs after iHSRs were 6.5 (range=1-19) and 3.0 (range $=0-7$ ) in the continuation group and the discontinuation group, respectively. As expected, the median number of cycles of PTs was significantly higher in the continuation 


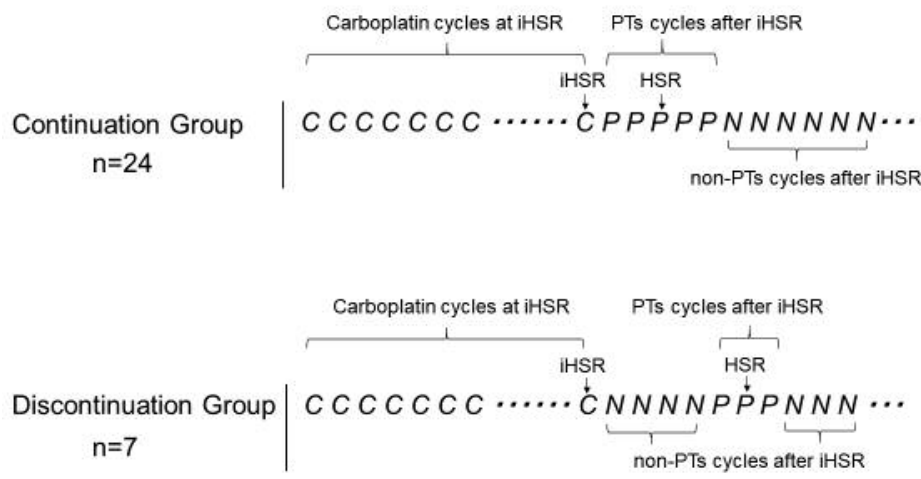

Figure 1. Models in the continuation and the discontinuation groups. PTs: Platinum agents that consist of carboplatin, cisplatin and nedaplatin; non-PTs: all chemotherapeutic agents except PTs; $C$ : one cycle of carboplatin administration; $P$ : one cycle of a PT administration; $N$ : one cycle of a non-PT administration; Dots: progression free interval; HSR: hypersensitivity reaction; iHSR: initial hypersensitivity reaction.

Table I. Patient characteristics.

\begin{tabular}{|c|c|c|c|}
\hline & $\begin{array}{l}\text { Continuation } \\
\text { group } \\
(n=24)\end{array}$ & $\begin{array}{l}\text { Discontinuation } \\
\text { group } \\
(\mathrm{n}=7)\end{array}$ & $p$-Value \\
\hline Age $(y)$, mean \pm SD & $58 \pm 10$ & $55 \pm 9$ & 0.45 \\
\hline FIGO Stage $(\%)$ & & & 0.33 \\
\hline I & $1(4)$ & $0(0)$ & \\
\hline II & $1(4)$ & $0(0)$ & \\
\hline III & $19(79)$ & $4(57)$ & \\
\hline IV & $3(13)$ & $3(43)$ & \\
\hline Histologic type (\%) & & & 0.27 \\
\hline Serous & $15(63)$ & $3(43)$ & \\
\hline Endometrioid & $3(13)$ & $1(14)$ & \\
\hline Clear cell & $3(13)$ & $0(0)$ & \\
\hline Mucinous & $1(4)$ & $0(0)$ & \\
\hline Others & $2(8)$ & $3(43)$ & \\
\hline $\begin{array}{l}\text { Carboplatin cycles at } \\
\text { iHSR, median (range) }\end{array}$ & $10(7-17)$ & $12(9-16)$ & 0.28 \\
\hline $\begin{array}{l}\text { Time to iHSR (months), } \\
\text { median (range) }\end{array}$ & $26.8(14.5-63.9)$ & $25.5(18.9-44.9)$ & 0.83 \\
\hline $\begin{array}{l}\text { Chemotherapy at the } \\
\text { time of iHSR }(\%)\end{array}$ & & & 1 \\
\hline Second line & $21(88)$ & $6(86)$ & \\
\hline Third line & $3(13)$ & $1(14)$ & \\
\hline Severity of iHSR (\%) & & & 0.15 \\
\hline G1 & $8(33)$ & $0(0)$ & \\
\hline $\mathrm{G} 2$ & $16(67)$ & $7(100)$ & \\
\hline G3-G5 & $0(0)$ & $0(0)$ & \\
\hline
\end{tabular}

SD: Standard deviation; FIGO: International Federation of Gynecology and Obstetrics; iHSR: initial hypersensitivity reaction; G1-G5: grade scale of the allergic reaction category of the Common Terminology Criteria for Adverse Events (CTCAE ver4.0).

group ( $p=0.048$ ). The median number of cycles of non-PTs and total cycles of chemotherapy agents following iHSRs, which were calculated by the addition of PTs and the non-
Table II. Treatment administration following iHSR.

\begin{tabular}{lccc}
\hline & $\begin{array}{c}\text { Continuation } \\
\text { group } \\
(\mathrm{n}=24)\end{array}$ & $\begin{array}{c}\text { Discontinuation } \\
\text { group } \\
(\mathrm{n}=7)\end{array}$ & $p$-Value \\
& & & \\
\hline $\begin{array}{l}\text { No. of cycles, } \\
\text { median (range) }\end{array}$ & $6.5(1-19)$ & $3.0(0-7)$ & 0.048 \\
$\quad$ PTs & $5.5(0-23)$ & $6.0(0-11)$ & 0.6 \\
$\quad$ Non-PTs & $10(2-32)$ & $9(0-18)$ & 0.55 \\
$\quad$ Total & & & \\
No. of regimens, & & & \\
median (range) & $2(1-4)$ & $1(0-2)$ & 0.009 \\
$\quad$ PTs & $1(0-4)$ & $1(0-3)$ & 0.64 \\
$\quad$ Non-PTs & $3(1-4)$ & $2(0-4)$ & 0.13 \\
Total & & & \\
\hline
\end{tabular}

iHSRs: Initial hypersensitivity reactions; PTs: platinum agents that consist of carboplatin, cisplatin and nedaplatin; non-PTs: all chemotherapeutic agents except PTs.

PTs, were not significantly different between the two groups. The median number of regimens with PTs following iHSRs were 2 (range $=1-4)$ and 1 (range $=0-2$ ) in the continuation group and the discontinuation group, respectively, with a significantly higher number of regimens in the continuation group $(p=0.009)$. The median number of regimens with nonPTs and total regimens were not significantly different between two groups.

Overall survival in two groups. OS was significantly longer in the continuation group compared to the discontinuation group. Median OS was 28.1 months [95\% confidence interval $(\mathrm{CI})=20.2-43.6]$ in the continuation group and 15.4 months $(95 \% \mathrm{CI}=1.7-27.5)$ in the discontinuation group $(p=0.018)$ (Figure 2). Multivariate analysis was performed 


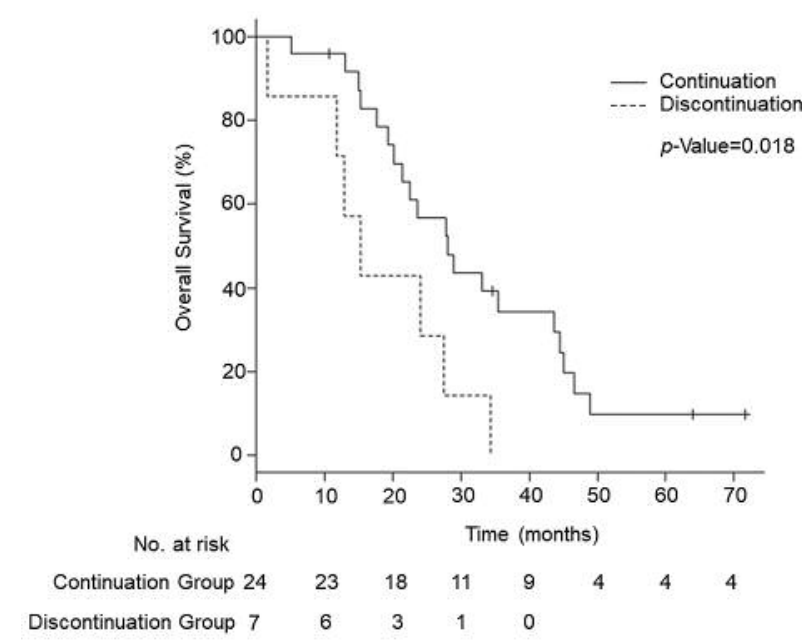

Figure 2. Kaplan-Meier curves of overall survival in the two groups. The solid line represents the continuation group and dashed line represents the discontinuation group. Vertical lines represent censoring.

using age ( $\leq 50$ versus $>50$ ), histologic type (serous versus others), and continued administration of PTs after iHSR as prognostic variables. Stage was excluded from prognostic variables as nearly all patients (29 of 31 ) included in the study represented FIGO stage III/IV (Table I). In the multivariate analysis, after adjusting for the prognostic variables, continuous administration of PTs following iHSR was associated with a significantly better OS (Hazard Ratio $=0.33,95 \% \mathrm{CI}=0.13-0.84, p=0.02$ ) (Table III).

Readministration of platinum agents in the continuation group. The method of readministration of platinum agents in the continuation group following the development of iHSR to carboplatin is shown in Table IV. In the continuation group, of the total number of 155 cycles of PTs readministered, 50 cycles (32\%) led to recurrent HSRs. The severity of the allergic reaction was G1 in 23, and G2 in 27 cycles. No reaction of G3 or greater occurred in any recurrent HSR case. The median number of recurrent HSRs following iHSRs was 1 (range=0-7) per patient. Readministration was performed after hospitalization in $89 \%$ of these cycles. One hundred nineteen cycles were readministered with extra preventive measures, including doubling of the administration time in 7 cycles, increased dose of anti-inflammatory medication in 53 cycles, or both being performed in 59 cycles. Despite the extra preventive measures, 43 cycles (36\%) led to recurrent HSR. Carboplatin, cisplatin and nedaplatin were readministered in 124, 13 and 18 cycles, respectively. All recurrent reactions were in response to carboplatin, while there were no reactions to cisplatin or nedaplatin.
Table III. Prognostic value by multivariate analysis for overall survival. HR: Hazard ratio; 95\% CI: 95\% confidence interval; PTs: platinum agents that consist of carboplatin, cisplatin and nedaplatin.

\begin{tabular}{lcc}
\hline & HR $(95 \% \mathrm{CI})$ & $p$-Value \\
\hline $\begin{array}{l}\text { Age } \\
\quad 550 v s .>50\end{array}$ & $1.32(0.59-2.93)$ & 0.49 \\
$\begin{array}{l}\text { Histologic type } \\
\quad \text { Serous } v s . \text { others }\end{array}$ & $0.43(0.20-0.95)$ & 0.037 \\
$\begin{array}{l}\text { Administration of PTs } \\
\quad \begin{array}{l}\text { Continuation } v s . \\
\text { discontinuation }\end{array}\end{array}$ & $0.33(0.13-0.84)$ & 0.02 \\
\hline
\end{tabular}

\section{Discussion}

The results of this study suggest that continuous administration of PTs may improve OS compared to discontinuation of PTs in patients with recurrent ovarian cancer who develop HSRs to carboplatin. We attribute the extension of OS in the continuation group to the greater number of cycles of PTs administered following iHSRs compared to the discontinuation group. Until now, only strategies investigating the methods for readministration of PTs have been reported. The findings of this study are the first to support the assumption that continuation of PTs could beneficially impact OS, despite the recurrence of an HSR, provided that the patient maintains sensitivity to these agents. This study was limited by the small cohort, however, these results could be helpful to consider the continuation of PTs when HSRs occur in recurrent ovarian cancer.

Our data demonstrate that, although HSRs recur at a high rate in patients receiving PT readministration, subsequent HSRs are unlikely to aggravate to a serious state. Recurrence of HSR in our case was observed regardless of preventive measures, but the reactions were all of G2 severity or lower and were remitted by symptomatic treatment, such as withdrawal of the drug and steroid administration. These findings support the validity of attempted readministration of PTs in patients who develop HSRs to carboplatin. Recently some studies reported that a skin test or germline BRCA1/2 mutation status seemed to be able to predict the development of HSRs to carboplatin $(15,16)$. Premedication and desensitization therapies, such as multistep dilution infusion, and extended infusion may also prevent recurrent HSRs and have been used successfully in many studies (10-12). Therefore, readministration of PTs can be made safe with appropriate surveillance during hospitalization in patients with a low predicted risk of recurrent HSRs and using appropriate preventive measures.

There are two limitations of the present study. First, the retrospective design necessarily lacks randomization. It is possible that the decision to continue or discontinue PTs was made for a reason that is not clear in the medical records. 
Table IV. Readministration of platinum agents in the continuation group (total 155 cycles). Situations and outcomes of readministration of platinum agents are presented as numbers.

\begin{tabular}{|c|c|c|c|}
\hline \multirow{2}{*}{ Setting of administration } & \multirow[t]{2}{*}{ No recurrent HSRs $(n=105)$} & \multicolumn{2}{|c|}{$\begin{array}{c}\text { Recurrent HSRs (n=50) } \\
\text { Severity }(\mathrm{G} 1 / \mathrm{G} 2 / \mathrm{G} 3-\mathrm{G} 5)\end{array}$} \\
\hline & & & \\
\hline Outpatient & 9 & 8 & $(1 / 7 / 0)$ \\
\hline Inpatient & 90 & 42 & $(22 / 20 / 0)$ \\
\hline Not available & 6 & 0 & \\
\hline \multicolumn{4}{|l|}{ Preventative measure } \\
\hline No extra preventative measures & 22 & 7 & $(1 / 6 / 0)$ \\
\hline Extra preventive measures & 76 & 43 & \\
\hline i) Extend the administration time & (4) & (3) & $(0 / 3 / 0)$ \\
\hline ii) Dose up of antiallergic medication & (40) & (13) & $(2 / 11 / 0)$ \\
\hline iii) Both & (32) & (27) & $(20 / 7 / 0)$ \\
\hline Not available & 7 & 0 & \\
\hline \multicolumn{4}{|l|}{ Platinum agent } \\
\hline Carboplatin & 74 & 50 & $(23 / 27 / 0)$ \\
\hline Cisplatin & 13 & 0 & \\
\hline Nedaplatin & 18 & 0 & \\
\hline
\end{tabular}

HSR: Hypersensitivity reaction; G1-G5: grade scale of the allergic reaction category of the Common Terminology Criteria for Adverse Events (CTCAE ver4.0).

Some cases might have been considered for continued PTs by the attending physicians because the HSR reactions were very light, or PTs were deemed highly effective. Additionally, some patients might have urged for or rejected continuing the platinum therapy for unknown reasons. However, there was no difference in the patients' backgrounds between the two groups and it is unlikely that bias was directly associated with the difference in outcomes.

The second limitation is that a G3 or more severe allergic reaction following repeated HSRs may not have appeared due to the small patient population. This may be a low frequency reaction. In fact, fatal cases of life-threatening HSRs have been described, regardless of initial or recurrent development. Death due to cisplatin readministration following HSR to carboplatin has also been reported $(6,17-19)$. These possibilities cannot be neglected. However, patients who developed HSRs three times or more were included in our study, and symptoms did not aggravate to a serious state, even after repeated recurrence. Ultimately, while severe HSRs may develop, they may occur at any time during the treatment. Thus, the best approach is to take appropriate countermeasures, as described above, from the start of treatment in patients with high risk of developing HSRs, such as those with platinum-sensitive recurrent ovarian cancer. When HSRs occur, the benefit of continuing PTs should be carefully considered, and readministration should be considered after taking preventive measures against a recurrence, using a surveillance system and preparing a symptomatic treatment for recurrence. Readministration of PTs should, however, clearly be discontinued when severe HSRs develop.
The results of this retrospective study show that continuation of PTs in patients with recurrent ovarian cancer who develop HSRs to carboplatin may contribute to improvement in their survival. Since HSRs are likely to recur, but unlikely to aggravate to a serious state, continuation of PTs may be safe with thorough preparation. Because the number of cases in this study is limited, establishment of an effective and safe treatment regimen for patients with ovarian cancer who develop HSRs will require accumulation of more data in larger studies.

\section{Conflicts of Interest}

None of the authors have a conflict of interest with regard to the study.

\section{Authors' Contributions}

Study design: CN, HT; Data collection: CN, HT, YN, TM, MI, YH, HK; Formal analysis; CN, HT, JSS; Resources: CN, HT, YN, TM, MI, YH, HK, HT, SN, SI, AO. CN was a major contributor in writing the manuscript and HT was a supervisor.

\section{References}

1 Bray F, Ferlay J, Soerjomataram I, Siegel RL, Torre LA, Jemal A: Global cancer statistics 2018: GLOBOCAN estimates of incidence and mortality worldwide for 36 cancers in 185 countries. CA Cancer J Clin 68(6): 394-424, 2018. PMID: 30207593. DOI: $10.3322 /$ caac. 21492 
2 Ozols RF, Bundy BN, Greer BE, Fowler JM, Clarke-Pearson D, Burger RA, Mannel RS, DeGeest K, Hartenbach EM, Baergen $\mathrm{R}$ and Gynecologic Oncology Group: Phase III trial of carboplatin and paclitaxel compared with cisplatin and paclitaxel in patients with optimally resected stage III ovarian cancer: A Gynecologic Oncology Group study. J Clin Oncol 21(17): 31943200, 2003. PMID: 12860964. DOI: 10.1200/JCO.2003.02.153

3 Bookman MA, Brady MF, McGuire WP, Harper PG, Alberts DS, Friedlander M, Colombo N, Fowler JM, Argenta PA, De Greest K, Mutch DG, Burger RA, Swart AM, Trimble EL, AssarioWinslow $\mathrm{C}$ and Roth LM: Evaluation of new platinum-based treatment regimens in advanced-stage ovarian cancer: a Phase III Trial of the Gynecologic Cancer Intergroup. J Clin Oncol 27(9): 1419-1425, 2009. PMID: 19224846. DOI: 10.1200/ JCO.2008.19.1684

4 Berek JS, Kehoe ST, Kumar L and Friedlander M: Cancer of the ovary, fallopian tube, and peritoneum. Int J Gynaecol Obstet 143(Suppl 2): 59-78, 2018. PMID: 30306591. DOI: 10.1002/ ijgo. 12614

5 Sun T and Li L: A cohort study of hypersensitivity reaction in patients with epithelial ovarian cancer treated with carboplatin. Int J Gynecol Cancer 29(3): 566-571, 2019. PMID: 30833442. DOI: $10.1136 / \mathrm{ijgc}-2018-000072$

6 Markman MD M, Kennedy A, Webster K, Elson P, Peterson G, Kulp B and Belinson J: Clinical features of hypersensitivity reactions to carboplatin. J Clin Oncol 17(4): 1141-1145, 1999. PMID: 10561172. DOI: 10.1200/JCO.1999.17.4.1141

7 Michikami H, Minaguchi T, Ochi H, Onuki M, Okada S, Matsumoto K, Satoh T, Oki A and Yoshikawa H: Safety and efficacy of substituting nedaplatin after carboplatin hypersensitivity reactions in gynecologic malignancies. J Obstet Gynaecol Res 39(1): 330-335, 2013. PMID: 22640034. DOI: 10.1111/j.1447-0756.2012.01893.x

8 Polyzos A, Tsavaris N, Kosmas C, Arnaouti T, Kalahanis N, Tsigris C, Giannopoulos A, Karatzas G, Giannikos L and Sfikakis PP: Hypersensitivity reactions to carboplatin administration are common but not always severe: a 10 -year experience. Oncology 61(2): 129-133, 2001. PMID: 11528251. DOI: $10.1159 / 000055363$

9 Koshiba H, Hosokawa K, Kubo A, Miyagi Y, Oada T, Miyagi Y, Wartanabe A and Honjo H: Incidence of Carboplatin-related hypersensitivity reactions in Japanese patients with gynecologic malignancies. Int J Gynecol Cancer 19(3): 460-465, 2009. PMID: 19407575. DOI: 10.1111/IGC.0b013e3181a1bf2e

10 Vetter MH, Khan A, Backers FJ, Bixel K, Cohn DE, Copeland LJ, Fowler JM, Salani R, Li Q and O'Malley DM: Outpatient desensitization of patients with moderate (high-risk) to severe platinum hypersensitivity reactions. Gynecol Oncol 152(2): 316321, 2019. PMID: 30503265. DOI: 10.1016/j.ygyno.2018.10.037

11 Altwerger G, Gressel GM, English DP, Nelson WK, Carusillo N, Silasi DA, Azodi M, Santin A, Schwartz PE and Ratner ES: Platinum desensitization on patients with carboplatin hypersensitivity: A single-institution retrospective study. Gynecol Oncol 144(1): 77-82, 2017. PMID: 27789084. DOI: 10.1016/j.ygyno.2016.09.027
12 O'Cearbhaill R, Zhou Q, Iasonos A, Hensley ML, Tew WP, Aghajanian C, Spriggs DR, Lichtman SM and Sabbatini PJ: The prophylactic conversion to an extended infusion schedule and use of premedication to prevent hypersensitivity reactions in ovarian cancer patients during carboplatin retreatment. Gynecol Oncol 116(3): 326-331, 2010. PMID: 19944454. DOI: 10.1016/ j.ygyno.2009.10.070

13 Callahan MB, Lachance JA, Stone RL, Kelsey J, Rice LW and Jazaeri AA: Use of cisplatin without desensitization after carboplatin hypersensitivity reaction in epithelial ovarian and primary peritoneal cancer. Am J Obstet Gynecol 197(2): 199.e14, 2007. PMID: 17689649. DOI: 10.1016/j.ajog.2007.04.044

14 Common Terminology Criteria for Adverse Events (CTCAE) Version 4.0, U.S. Department of health and human services, Available at http://www.eortc.be/services/doc/ctc/CTCAE_4.03_ 2010-06-14_QuickReference_5x7.pdf (Last accessed on 31st July 2019)

15 Lax T, Long A and Banerji A: Skin testing in the evaluation and management of carboplatin-related hypersensitivity reactions. J Allergy Clin Immunol Pract 3(6): 856-862, 2015. PMID: 26432514. DOI: $10.1016 /$ j.jaip.2015.07.003

16 Altwerger G, Florsheim EB, Menderes G, Black J, Schwab C, Gressel GM, Nelson WK, Carusillo N, Passante T, Huang G, Likouhi B, Azodi M, Silasi DA, Santin A, Schwartz PE and Ratner ES: Impact of carboplatin hypersensitivity and desensitization on patients with recurrent ovarian cancer. J Cancer Res Clin Oncol 144(12): 2449-2456, 2018. PMID: 30255380. DOI: 10.1007/s00432-018-2753-y.

17 Kook H, Kim KM, Choi SH, Choi BS, Kim HJ, Chung SY, Choi SJ and Hwang TJ: Life-threatening carboplatin hypersensitivity during conditioning for autologous PBSC transplantation: successful rechallenge after desensitization. Bone Marrow Transpl 21(7): 727-729, 1998. PMID: 9578315. DOI: 10.1038/ sj.bmt. 1701161

18 Watanabe Y, Nakai H, Ueda H, Nozaki K and Hoshiai H: Carboplatin hypersensitivity induced by low-dose paclitaxel/ carboplatin in multiple platinum-treated patients with recurrent ovarian cancer. Int J Gynecol Cancer 15(2): 224-227, 2005. PMID: 15823103. DOI: 10.1111/j.1525-1438.2005. 15207.x

19 Dizon DS, Sabbatini PJ, Aghajanian C, Hensley ML and Spriggs DR: Analysis of patients with epithelial ovarian cancer or fallopian tube carcinoma retreated with cisplatin after the development of a carboplatin allergy. Gynecol Oncol 84(3): 378382, 2002. PMID: 11855873. DOI: 10.1006/gyno.2001.6519
Received July 10, 2019

Revised August 2, 2019 Accepted August 5, 2019 